\title{
An Explosive Scaling Law for Nonlinear Magnetic Reconnection and Its Insensitivity to Microscopic Scales*)
}

\author{
Makoto HIROTA \\ Institute of Fluid Science, Tohoku University, Sendai, Miyagi 980-8577, Japan
}

(Received 11 January 2017 / Accepted 16 February 2017)

\begin{abstract}
The nonlinear phase of magnetic reconnection is investigated by numerically solving a gyrofluid model. The scaling law for the explosive reconnection rate, which has been recently derived for an ideal two-fluid model [Hirota et al., Phys. Plasmas 22, 052114 (2015)], is found to consistently hold when either the ion-sound gyroradius $\rho_{\mathrm{S}}$ or the ion gyroradius $\rho_{\mathrm{i}}$ is comparable to the electron skin depth $d_{\mathrm{e}}$, even in the presence of finite resistivity $\eta$. In this explosive phase, a local X-shaped current layer is spontaneously generated, in which the reconnection speed is closely related to the macroscopic shape of the layer and is almost independent of the layer width. The reconnection speed is therefore insensitive to the size of the microscopic scales, $\rho_{\mathrm{S}}, \rho_{\mathrm{i}}, d_{\mathrm{e}}$ and $\eta$. On the other hand, in the cold plasma limit, where $\rho_{\mathrm{S}}=\rho_{\mathrm{i}}=0$, the intermittent acceleration of the reconnection speed is caused by the plasmoid instability. This also seems to be explosive on average, but the rate always falls below the explosive scaling law. The reconnection time extrapolated from this scaling law is shown to be fast enough to explain the time scale of solar flares.
\end{abstract}

(c) 2017 The Japan Society of Plasma Science and Nuclear Fusion Research

Keywords: magnetic reconnection, explosive instability, gyrofluid model, plasmoid instability, solar flare

DOI: $10.1585 /$ pfr.12.1401010

\section{Introduction}

Fast magnetic reconnection in a collisionless plasma has drawn considerable attention from plasma physicists for many years. In resistive magnetohydrodynamics (MHD) theory, the Sweet-Parker model [1] provided a basic understanding of steady reconnection, but the estimated reconnection time is too slow to explain solar flares. In subsequent investigations, the elongated current sheet of the Sweet-Parker model was found to be unstable to, what is now called, the plasmoid instability, when the resistivity is sufficiently small [2,3]. Many investigations [4-6] currently expect that subsequent unsteady or stochastic processes may account for fast magnetic reconnection.

On the other hand, it is also suggested that the resistive MHD model needs to be extended to more general twofluid or gyrofluid model for the purpose of studying collisionless reconnections, in which the microscopic scales, such as the skin depth and the gyroradius, can be more important than resistivity. The Global Environment Modeling (GEM) Reconnection Challenge [7] reports that the reconnection speed is significantly enhanced when the Hall current term ( $\propto d_{i}$, the ion skin depth) is included. In the context of magnetized plasma models in a strong guide field, the effects of the ion-sound gyroradius $\rho_{\mathrm{S}}=\sqrt{\beta_{\mathrm{e}}} d_{\mathrm{i}}$ and the ion gyroradius $\rho_{\mathrm{i}}=\sqrt{\beta_{\mathrm{i}}} d_{\mathrm{i}}$ lead to similar enhancements of the reconnection speed. (Here, $\beta_{\mathrm{e}, \mathrm{i}}$ are the electron's and ion's beta values). Many simulation results [8-13] confirm

author'se-mail: hirota@dragon.ifs.tohoku.ac.jp

*) This article is based on the invited talk at the 33rd JSPF Annual Meeting (2016, Tohoku). that when $\rho_{\mathrm{S}}$ and $\rho_{\mathrm{i}}$ are comparable to the electron skin depth $d_{\mathrm{e}}$, the reconnection rate is nonlinearly accelerated by an X-shaped current layer (which is only visually similar to the Petschek model [14]). Using an ideal two-fluid model $\left(d_{\mathrm{e}}=\rho_{\mathrm{S}} \neq 0, \rho_{\mathrm{i}}=0\right.$, and no resistivity $\eta=0$ ), our recent work [15] has shown that this X-shaped layer is actually localized around the reconnection point and that this spatially concentrated reconnection is responsible for persistent nonlinear acceleration which, moreover, appears to be explosive.

In this paper, we numerically solve a gyrofluid model that includes the four microscale parameters $\left(d_{\mathrm{e}}, \rho_{\mathrm{S}}, \rho_{\mathrm{i}}, \eta\right)$, and we investigate whether or not the reconnection becomes explosive, by focusing on its nonlinear phase. Because of the limitation of computational power, it is not possible to survey every combination of the four parameters $\left(d_{\mathrm{e}}, \rho_{\mathrm{S}}, \rho_{\mathrm{i}}, \eta\right)$. Nevertheless, we show that almost the same explosive reconnection speed as found in Ref. [15] is obtained whenever a local X-shaped layer emerges, even though the layer width varies, depending on the values of the microscale parameters. This explosive scaling law may therefore be universal in that it is insensitive to the microscale physics.

In the limit of a cold plasma, where $\rho_{\mathrm{S}}=\rho_{\mathrm{i}}=0$, the plasmoid instability occurs instead of the generation of a single X-shaped layer. While the reconnection speed does fluctuate stochastically, we show below that it tends to follow the explosive scaling law. Hence, reconnection is likely to be explosive on average even in this case.

Finally, we predict the reconnection (or collapse) time 
of the plasma by applying this explosive scaling law to a system of arbitrary size. We show that the estimated time for reconnection is fast enough to explain the observed time scale of solar flares.

\section{Magnetized Low- $\beta$ Plasma Model}

We consider the following reduced gyrofluid model in Cartesian coordinates [10,16];

$$
\begin{aligned}
& \frac{\partial n_{\mathrm{e}}}{\partial t}+\left[\phi, n_{\mathrm{e}}\right]=\left[\psi, \nabla^{2} \psi\right] \\
& \frac{\partial\left(\psi-d_{\mathrm{e}}^{2} \nabla^{2} \psi\right)}{\partial t}+\left[\phi, \psi-d_{\mathrm{e}}^{2} \nabla^{2} \psi\right] \\
& =\rho_{\mathrm{S}}^{2}\left[n_{\mathrm{e}}, \psi\right]+\eta \nabla^{2}\left(\psi-\psi_{\text {eq. }}\right), \\
& n_{\mathrm{e}}-n_{\mathrm{i} 0}=\frac{\nabla^{2}}{1-\rho_{\mathrm{i}}^{2} \nabla^{2}} \phi
\end{aligned}
$$

where $\boldsymbol{v}=\boldsymbol{e}_{z} \times \nabla \phi(x, y, t)$ is the (ion's) velocity field, $\boldsymbol{B}=\sqrt{\mu_{0} m_{\mathrm{i}} n_{\mathrm{i} 0}} \nabla \psi(x, y, t) \times \boldsymbol{e}_{z}+B_{0} \boldsymbol{e}_{z}$ is the magnetic field and $[f, g]=(\nabla f \times \nabla g) \cdot \boldsymbol{e}_{z}$ (here, $\mu_{0}$ is the magnetic permeability, and $m_{\mathrm{i}}$ is the ion mass). We assume that the ion density $n_{\mathrm{i} 0}$, ion and electron temperatures $T_{\mathrm{i}, \mathrm{e}}$ and guide field $B_{0}$ are spatially uniform. Equations (1) through (3) can be derived approximately for a low- $\beta$ plasma, where $\beta=\mu_{0} n_{\mathrm{i} 0}\left(T_{\mathrm{i}}+T_{\mathrm{e}}\right) / B_{0}^{2} \ll 1$, while retaining the scales of the ion-sound gyroradius $\rho_{\mathrm{S}}=\sqrt{m_{\mathrm{i}} T_{\mathrm{e}} /\left(e^{2} B_{0}^{2}\right)}$ and ion gyroradius $\rho_{\mathrm{i}}=\sqrt{m_{\mathrm{i}} T_{\mathrm{i}} /\left(e^{2} B_{0}^{2}\right)}(e$ is the electron charge). The electron skin depth $d_{\mathrm{e}}=\sqrt{m_{\mathrm{e}} /\left(\mu_{0} n_{0} e^{2}\right)}$ (where $m_{\mathrm{e}}$ is the electron mass) and the magnetic diffusion coefficient $\eta$ represent the effects of electron inertia and electrical resistivity, respectively; both enable magnetic reconnection. We remark that the Padé approximation has been used in Eq. (3) to simplify the gyro-averaging operator. References $[12,17,18]$ discuss more general two-fluid and gyrofluid models in the context of magnetic reconnection.

In the resistive dissipation term in Ohm's law (2), we have artificially subtracted $\psi$ eq. from $\psi$, so that the equilibrium field $\psi_{\text {eq. }}$ does not change during the lengthy linear phase of the resistive tearing instability. We specifically choose the equilibrium state to be given by

$$
\phi_{\text {eq. }} \equiv 0, \quad \psi_{\text {eq. }}(x)=\psi_{0} \cos \frac{2 \pi x}{L_{x}},
$$

in a domain $\left[-L_{x} / 2, L_{x} / 2\right] \times\left[-L_{y} / 2, L_{y} / 2\right]$ that is periodic in both the $x$ and $y$ directions. For a linear perturbation with a wavenumber $k_{y}=2 \pi / L_{y}$ in the $y$ direction, the tearing index $\Delta^{\prime}$ can be calculated analytically (see p.103 of Ref. [19]). The result may be written as a function of the aspect ratio $a=2 \pi / k_{y} L_{x}=L_{y} / L_{x}$;

$$
\Delta^{\prime}(a)=\frac{4 \pi}{L_{x}} \sqrt{1-a^{-2}} \tan \left[\frac{\pi}{2} \sqrt{1-a^{-2}}\right] .
$$

When $L_{y} / L_{x}>1$, the equilibrium state is linearly unstable to the tearing mode with the wavenumber $k_{y}$. Then magnetic reconnection occurs at $x=0, \pm L_{x} / 2$ where $B_{y}$ becomes zero. We denote by $\epsilon(t)$ the displacement of the strongest magnetic field lines initially located at $x=$ $\pm L_{x} / 4$; these two field lines behave in a similar way because of the parity of the tearing mode. This quantity $\epsilon(t)$ will be regarded as the amplitude of the tearing mode as long as the mode pattern is coherent.

The linear growth rate $\gamma_{\mathrm{L}}$ of the tearing instability has been well-investigated by the asymptotic matching method [20]. When it becomes a maximum with respect to $k_{y}$, we typically find $\delta_{\text {in }} \Delta^{\prime} \sim 1$, where $\delta_{\text {in }}$ is the width of the (outermost) inner layer, which depends on $\left(d_{\mathrm{e}}, \rho_{\mathrm{S}}, \rho_{\mathrm{i}}, \eta\right)$. Since we have $\delta_{\text {in }} \ll L_{x}$ in many situations, we are interested in the case of large $\Delta^{\prime}\left(\sim 1 / \delta_{\text {in }} \gg 1 / L_{x}\right)$ or, equivalently, large aspect ratio $a$, and hence we may use the approximation,

$$
\Delta^{\prime}(a) \simeq 16 \frac{a^{2}}{L_{x}} \text { for } a \gg 1 .
$$

In general, one finds exponential growth $\epsilon(t) \propto e^{\gamma_{L} t}$ only in the linear phase; that is only when $\epsilon$ is much smaller than $\delta_{\text {in }}$ or any other microscopic scale. For small aspect ratios, where $\Delta^{\prime} \ll 1 / \delta_{\text {in }}$, the growth of $\epsilon$ is likely to be saturated below the level of $\delta_{\text {in }}$ (a survey of the dependence on $\Delta^{\prime}$ is given in Ref. [15] for an ideal two-fluid case). Conversely, in the large- $\Delta^{\prime}$ case $\left(\Delta^{\prime} \gtrsim 1 / \delta_{\text {in }}\right)$, the growth will not saturate, since there is no stabilizing mechanism in our problem. In fact, many simulation results confirm an acceleration of the growth rate in the strongly nonlinear phase, where $\epsilon>\delta_{\text {in. }}$. Generally speaking, two types of nonlinear phases are observed in the simulations, depending on whether or not the effects of the finite gyroradii $\rho_{\mathrm{S}}$ and $\rho_{\mathrm{i}}$ are negligible. In the next section, we discuss these features in detail, by reproducing them with our numerical code.

\section{Numerical Observation of Nonlin- ear Phases}

Our numerical code directly solves Eqs. (1)-(3) using Fourier spectral methods in both the $x$ - and $y$ directions and using forth-order Runge-Kutta method for time stepping. In the following, we use the normalization $\left(\hat{d}_{\mathrm{e}}, \hat{\rho}_{\mathrm{S}}, \hat{\rho}_{\mathrm{i}}, \hat{\epsilon}\right)=\left(d_{\mathrm{e}} / L_{x}, \rho_{\mathrm{S}} / L_{x}, \rho_{\mathrm{i}} / L_{x}, \epsilon / L_{x}\right)$ for brevity.

First, if we include only electron inertia $\hat{d}_{\mathrm{e}}=0.01$ in the cold and collisionless limit $\left(\rho_{\mathrm{S}}=\rho_{\mathrm{i}}=\eta=0\right)$, the width of the inner layer is simply $\delta_{\text {in }} \simeq d_{\mathrm{e}}$. Figure 1 shows that elongation of the current sheet occurs in the early nonlinear phase, where $\hat{\epsilon}=0.01$ to 0.03 , in agreement with Ref. [21]. However, we find that such the SweetParker-like current sheet becomes secondarily unstable to the plasmoid instability in the strongly nonlinear phase, where $\hat{\epsilon} \gtrsim 0.04$. Smaller magnetic islands (corresponding to plasmoids) are generated intermittently and then ejected up and down along the $y$-axis. Although this plasmoid instability is well-known to occur in resistive MHD simulations [2,3], it also seems to be true for collisionless 

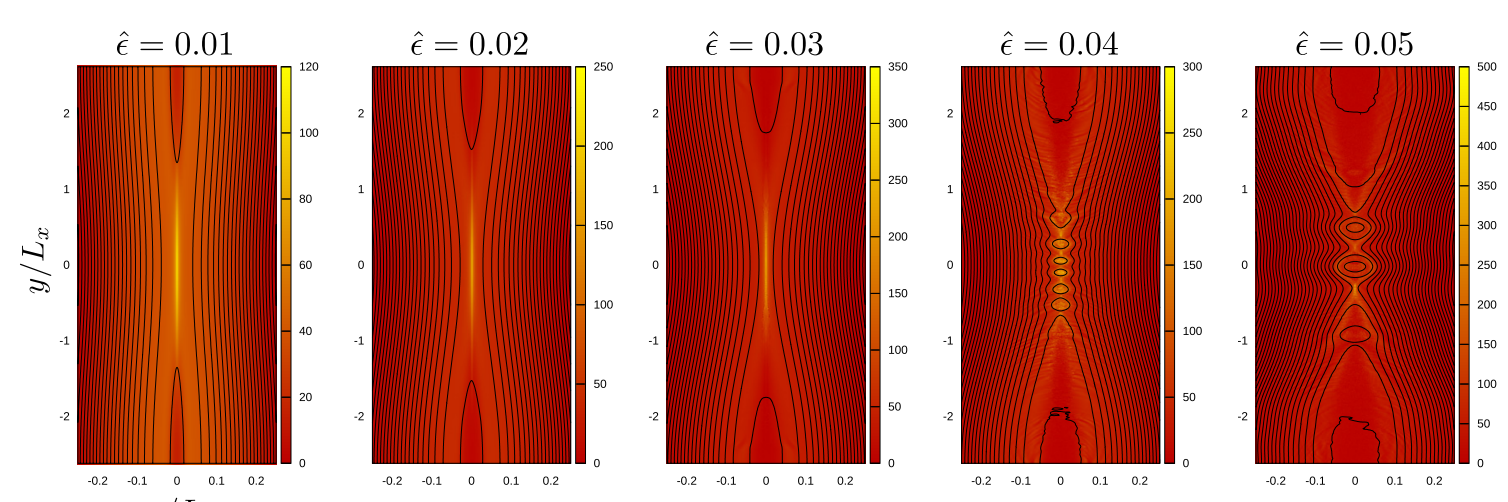

Fig. 1 Contours of $\psi$ (solid lines) and current distribution $\nabla^{2} \psi$ (color) for a case with $\hat{d}_{\mathrm{e}}=0.01, \rho_{\mathrm{S}}=\rho_{\mathrm{i}}=\eta=0$ and $L_{y} / L_{x}=5.24$ $\left(\Delta^{\prime}=427 / L_{x}\right)$, where the displacement $\hat{\epsilon}(t)$ grows monotonically and the snapshots are taken at times when $\hat{\epsilon}=0.01$ to 0.05 .
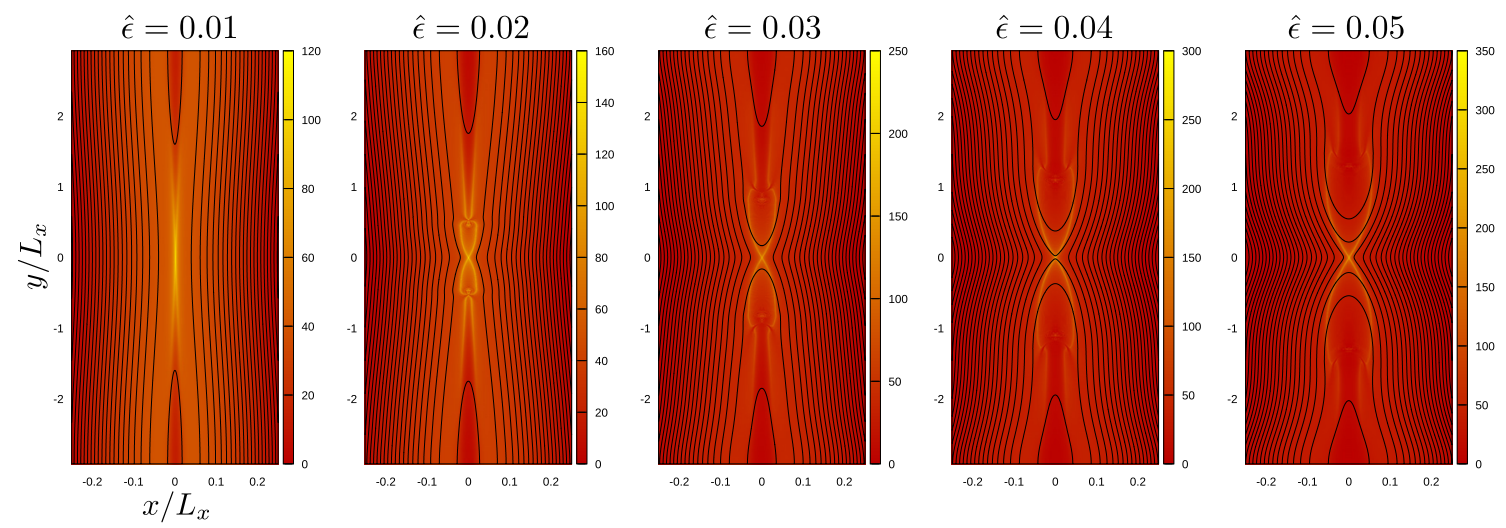

Fig. 2 Contours of $\psi$ (solid lines) and current distribution $\nabla^{2} \psi$ (color) for the case $\hat{d}_{\mathrm{e}}=\hat{\rho}_{\mathrm{S}}=0.01, \rho_{\mathrm{i}}=\eta=0$ and $L_{y} / L_{x}=5.84$ $\left(\Delta^{\prime}=534 / L_{x}\right)$, where the displacement $\hat{\epsilon}(t)$ grows monotonically and the snapshots are taken at times when $\hat{\epsilon}=0.01$ to 0.05 .
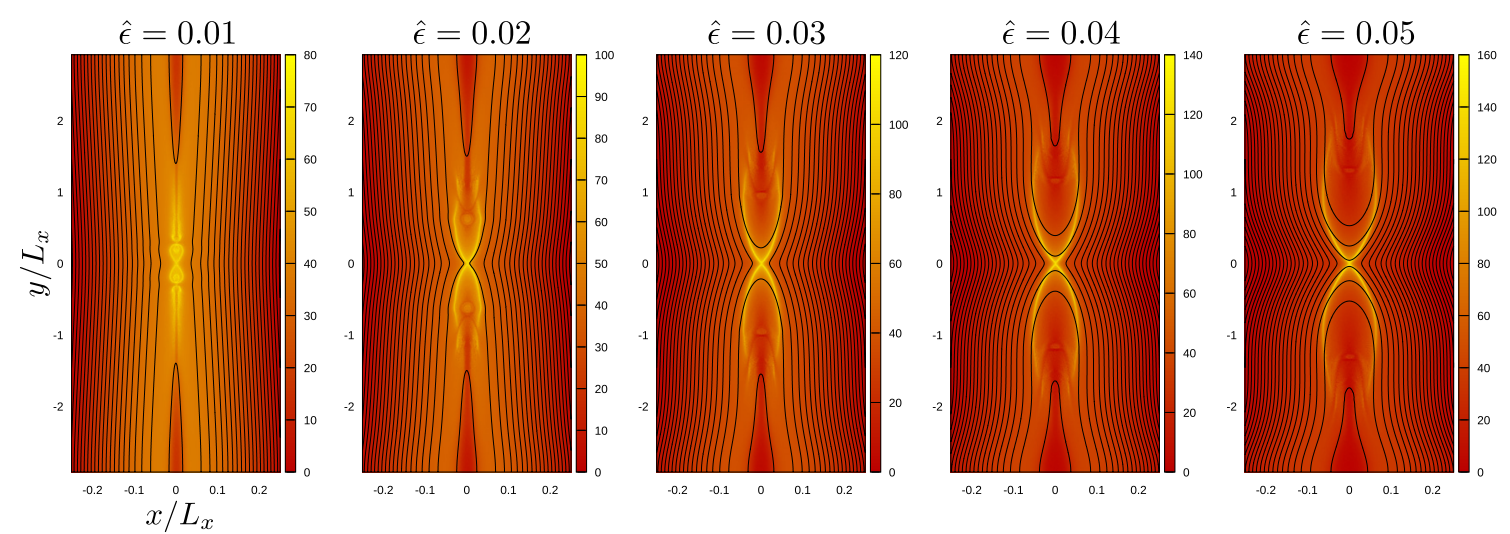

Fig. 3 Contours of $\psi$ (solid lines) and current distribution $\nabla^{2} \psi$ (color) for the case $\hat{d}_{\mathrm{e}}=\hat{\rho}_{\mathrm{i}}=0.01, \rho_{\mathrm{S}}=\eta=0$ and $L_{y} / L_{x}=5.84$ $\left(\Delta^{\prime}=534 / L_{x}\right)$, where the displacement $\hat{\epsilon}(t)$ grows monotonically and the snapshots are taken at times when $\hat{\epsilon}=0.01$ to 0.05 .

reconnection $\left(d_{\mathrm{e}} \neq 0, \eta=0\right)$. Our previous work [22] (and Ref. [21] as well) overlooked this secondary instability because the resolution for the $y$-direction was up to 200 Fourier modes, and we imposed a numerical viscosity to stabilize the finite-difference scheme employed in the $x$ direction. In the present paper, we obtain the result shown in Fig. 1 using a resolution of $4096 \times 4096$ and a similar onset of plasmoid instability is observed even when the resolution is increased to $8192 \times 8192$.

However, the nonlinear phase looks totally different when either $\rho_{\mathrm{S}}$ or $\rho_{\mathrm{i}}$ is comparable to $d_{\mathrm{e}}$. Figure 2 shows the case of $\hat{d}_{\mathrm{e}}=\hat{\rho}_{\mathrm{S}}=0.01$ and $\rho_{\mathrm{i}}=\eta=0$ (i.e., hot 
electrons $\beta_{\mathrm{e}}=m_{\mathrm{e}} / m_{\mathrm{i}}$ and cold ions $\left.\beta_{\mathrm{i}}=0\right)$ ). In this case, we find an $\mathrm{X}$-shaped current sheet to be generated nonlinearly, and we obtain an accelerated reconnection, as found in earlier works $[8,11,12]$. Our recent work [15] shows that this X-shaped layer emerges locally near the reconnection point (as shown in the panel with $\hat{\epsilon}=0.02$ in Fig. 2) and then expands rapidly and explosively.

Figure 3 shows the case of $\hat{d}_{\mathrm{e}}=\hat{\rho}_{\mathrm{i}}=0.01$ and $\rho_{\mathrm{S}}=\eta=0$ (i.e., hot ions with $\beta_{\mathrm{i}}=m_{\mathrm{e}} / m_{\mathrm{i}}$ and cold electrons with $\beta_{\mathrm{e}}=0$ ). A similar situation has already been studied in Ref. [10,12], and the effect of $\rho_{\mathrm{i}}$ was found to be similar to that of $\rho_{\mathrm{S}}$ so far as the nonlinear reconnection rate is concerned. Our simulation results shown in Fig. 3 additionally confirm that the $\mathrm{X}$-shaped layer is again localized in the nonlinear phase. The roles of $\rho_{\mathrm{i}}$ (Fig. 3) and $\rho_{\mathrm{S}}$ (Fig. 2) are slightly different, in that the small X-shaped layer shows up a little earlier and the layer width is a little wider in the former case than in the latter.

\section{An Explosive Scaling Law}

In order to distinguish whether magnetic reconnection is explosive or not, we first define a theoretical model that serves to illustrate the situation.

As a simplified picture, let us consider the equation of motion:

$$
\ddot{\boldsymbol{x}}=-\nabla U(\boldsymbol{x}),
$$

for a mass point $\boldsymbol{x}(t)$ in a potential $U$. Suppose that $U$ has a linearly-unstable equilibrium point $\boldsymbol{x}_{\mathrm{e}}$, at which $\nabla U\left(x_{\mathrm{e}}\right)=0$. In this case, a linear instability will occur if the displacement $\epsilon(t)=\left|\boldsymbol{x}(t)-\boldsymbol{x}_{\mathrm{e}}\right|$ is sufficiently small at $t=0$. As $t$ increases, $\epsilon$ continues to grow as far as $U$ decreases. Using the energy conservation law, the velocity at a position $\boldsymbol{x}$ is determined by the potential change $\delta U(\boldsymbol{x})=U(\boldsymbol{x})-U\left(\boldsymbol{x}_{\mathrm{e}}\right)$ as follows.

$$
\dot{\epsilon}=|\dot{x}|=\sqrt{-2 \delta U(\boldsymbol{x})} .
$$

The growth of $\epsilon$ is mathematically said to be explosive if it diverges $(\epsilon \rightarrow \infty)$ in a finite time. A typical example is given by

$$
\dot{\epsilon}=\frac{\epsilon^{s}}{\tau \epsilon_{0}^{s-1}}, \quad \epsilon(0)=\epsilon_{0},
$$

which has an explosive solution if $s>1$,

$$
\epsilon(t)=\left(\frac{1}{1-(s-1) t / \tau}\right)^{\frac{1}{s-1}} \epsilon_{0} .
$$

For this case, no matter how small the initial condition $\epsilon_{0}$ is, $\epsilon$ blows up in a finite time $\sim \tau$ (unless $s$ is too close to 1 ). In reality, however, $\epsilon$ cannot become infinity and the explosive growth often terminates because of finite system size $L$ or finite values of the free energy $U\left(x_{\mathrm{e}}\right)$. Consequently, it is more realistic to assume that there is some characteristic small scale $d$ that separates the linear and nonlinear phases:

$$
-\delta U(\epsilon)=\left\{\begin{array}{ll}
\frac{d^{2}}{2 \tau_{\mathrm{L}}^{2}}\left(\frac{\epsilon}{d}\right)^{2}\left[1+O\left(\frac{\epsilon}{d}\right)\right] & \text { for } \epsilon \ll d \\
\frac{d^{2}}{2 \tau_{\mathrm{N}}^{2}}\left(\frac{\epsilon}{d}\right)^{2 s}\left[1+O\left(\frac{d}{\epsilon}\right)\right] & \text { for } d \ll \epsilon \ll L
\end{array},\right.
$$

where again $s>1$. Then, the linear instability $\epsilon \propto e^{t / \tau_{\mathrm{L}}}$ occurs initially when $\epsilon \ll d$. After $\epsilon$ exceeds $d$, the amplitude grows explosively until it is eventually bounded by the system size $L$. This nonlinear phase is observed as an abrupt collapse of the equilibrium state into a lower energy state. This event occurs in a finite time $\sim \tau_{\mathrm{N}}$ no matter how much smaller $d$ is than $L$ (although $\tau_{\mathrm{N}}$ may not be independent of $d$ ).

Now, let us apply this example of explosive behavior to the case of magnetic reconnection. We accomplish this by replacing the small scale $d$ by the inner layer width $\delta_{\text {in }}$ and the potential $U$ by the magnetic energy $W=$ $(1 / 2) \int|\nabla \psi|^{2} d x d y$. The following discussion is a brief review of our previous theory [15], but here we greatly simplify the logic by ignoring the microscale physics inside the current layer.

First, we assume that the nonlinear acceleration phases, such as shown in Fig. 2 and Fig. 3, can be schematically modeled as shown in Fig. 4. We take the X-shaped current layer (denoted by the red lines) to be localized in a box with dimensions $[-\sigma, \sigma] \times\left[-l_{y}, l_{y}\right]$, and we assume the layer width (presumably $\sim \delta_{\text {in }}$ ) to be negligible in the nonlinear phase: $\delta_{\text {in }} \ll \epsilon \leq \sigma \ll L_{x} / 4$.

We also assume that the external solution at $x \in$ $\left[-L_{x} / 4,-\sigma\right] \cup\left[\sigma, L_{x} / 4\right]$ can be approximated by $\psi=$ $\psi_{\text {eq. }}+\psi_{\mathrm{L}}$ where $\psi_{\mathrm{L}}=-\boldsymbol{\xi} \cdot \nabla \psi_{\text {eq. }}$ is the linear ideal MHD eigenfunction, as in the conventional linear tearing theory, and the displacement field $\boldsymbol{\xi}$ is normalized by $\epsilon=\mid \boldsymbol{\xi}(x=$ $\left.\pm L_{x} / 4, y=0\right) \mid$ in accordance with the definition of $\epsilon$. However, the effective wavelength $\Lambda_{y}$ of the displacement is allowed to be less than $L_{y}$ so as to represent a local reconnection (that is, the shaded areas in Fig. 4 are almost immobile). For given $\epsilon$ and $\Lambda_{y}$, we can determine $\sigma$ by using $\Delta^{\prime}$,

$$
\sigma=\epsilon\left(\frac{2}{\sigma \Delta^{\prime}}+1\right), \quad \Delta^{\prime} \simeq 16 \frac{\Lambda_{y}^{2}}{L_{x}^{3}},
$$

where we have used Eq. (6), assuming $\Lambda_{y} / L_{x} \gg 1$. The incompressibility of the external solution requires

$$
\frac{\sigma}{\epsilon}=\frac{\Lambda_{y}}{4 l_{y}}
$$

By assuming $\Lambda_{y} / l_{y} \gg 1$, these relations lead to

$$
\sigma \simeq \frac{L_{x}^{3} l_{y}}{2 \Lambda_{y}^{3}} \simeq \frac{1}{2}\left(\frac{L_{x}^{3}}{8 l_{y}^{2}}\right)^{1 / 4} \epsilon^{3 / 4} .
$$

The most efficient decrease of the magnetic energy (i.e., the potential energy) is expected when $\psi$ becomes 
nearly flat (or constant) inside the magnetic island. The magnetic energy in the region $[-\sigma, \sigma]$ is then negligible to leading order. In other words, the magnetic energy that flows into this area disappears due to magnetic reconnection. We can estimate this decrease of magnetic energy by using only the external solution at $x= \pm \sigma$,

$$
\begin{aligned}
\delta W & =-\frac{\Lambda_{y}}{2}\left[\left.\psi_{\mathrm{L}} \psi_{\mathrm{L}}^{\prime}\right|_{\substack{x=\sigma \\
y=0}}-\left.\psi_{\mathrm{L}} \psi_{\mathrm{L}}^{\prime}\right|_{\substack{x=-\sigma \\
y=0}}\right] \\
& =-\Lambda_{y} \tau_{\mathrm{H}}^{-2} \frac{\sigma^{3}}{\frac{2}{\sigma \Delta^{\prime}}+1} \\
& \simeq-4 l_{y} \tau_{\mathrm{H}}^{-2} \sigma^{3}
\end{aligned}
$$

where $\tau_{\mathrm{H}}^{-1}=\psi_{\mathrm{eq}}^{\prime \prime}||_{x=0}=\left(2 \pi / L_{x}\right)^{2} \psi_{0}$.

Since the inflow velocity (in the $x$-direction) toward the box region $[-\sigma, \sigma] \times\left[-l_{y}, l_{y}\right]$ is $\dot{\sigma}$, the outflow velocity (in the $y$-direction) is expected to be $\dot{\sigma} l_{y} / \sigma$ according to the incompressibility of the flow (see Fig. 4). The kinetic energy of the outflow within the magnetic island is therefore roughly given by

$$
\delta K \simeq 2 l_{y}^{2} L_{y} \frac{\dot{\sigma}^{2}}{\sigma} .
$$

Using the energy conservation law $\delta K+\delta W=0$, we can calculate $\dot{\sigma}$ as

$$
\dot{\sigma} \simeq \sqrt{\frac{2}{L_{y} l_{y}}} \tau_{\mathrm{H}}^{-1} \sigma^{2},
$$

which indicates that the smaller the value of $l_{y}$, the faster magnetic reconnection occurs. However, $l_{y}$ must satisfy $\sigma \ll l_{y}$ in order for (14) not to contradict the assumption $\Lambda_{y} / L_{x} \gg 1$. Thus, we expect $l_{y}$ to be of the order of $l_{y} \sim$ $L_{x}$, which is indeed plausible from Fig. 2 and Fig. 3. The

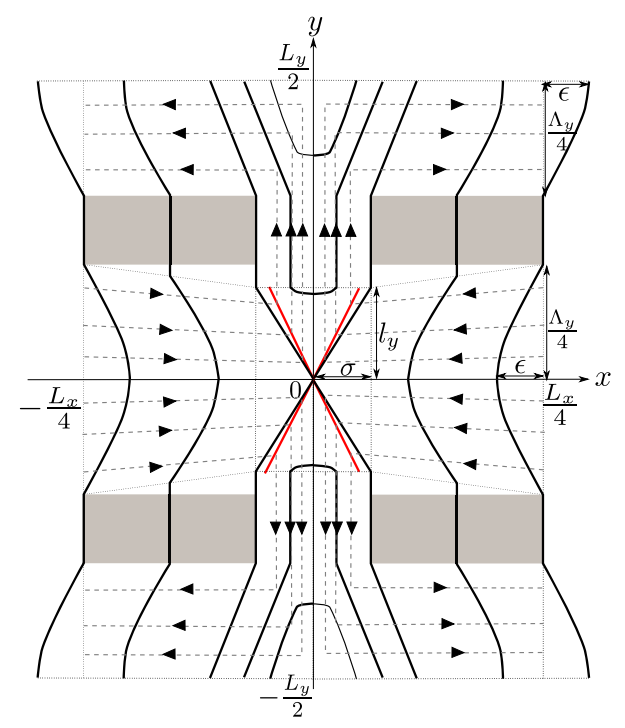

Fig. 4 Model of local reconnection (solid lines: magnetic field lines, dashed lines: streamlines, red lines: current layers). explosive growth (19) of $\sigma$ can then be rewritten in terms of $\epsilon$ and $\delta W$ as

$$
\begin{aligned}
& \frac{\dot{\epsilon}}{L_{x}} \simeq \tau_{\mathrm{H}}^{-1}\left(\frac{L_{x}}{L_{y}}\right)^{1 / 2}\left(\frac{\epsilon}{L_{x}}\right)^{7 / 4}, \\
& \frac{-\delta \dot{W}}{W_{0}} \simeq \tau_{\mathrm{H}}^{-1}\left(\frac{L_{x}}{L_{y}}\right)^{1 / 6}\left(\frac{-\delta W}{W_{0}}\right)^{4 / 3},
\end{aligned}
$$

where $\delta W$ is normalized by the initial magnetic energy $W_{0}=\left.W\right|_{t=0}=\tau_{\mathrm{H}}^{-2} L_{x}^{3} L_{y} /\left(16 \pi^{2}\right)$.

\section{Comparison with Simulation Re- sults}

In this section, we investigate how well the theoretical predictions (20) and (21) agree with the numerical results.

In Fig. 5, we compare cases with several different values of $d_{\mathrm{e}}$ and $\rho_{\mathrm{S}}$, keeping $\rho_{\mathrm{i}}=\eta=0$ and $L_{y} / L_{x}=5.84$ (the aspect ratio is chosen such that the maximum linear growth rate appears when $\left.\hat{d}_{\mathrm{e}}=\hat{\rho}_{\mathrm{S}}=0.005\right)$. As already shown in Ref. [15], the transition from the linear phase $\dot{\hat{\epsilon}}=\gamma_{\mathrm{L}} \hat{\epsilon}$ to the nonlinear one (20) is visible in Fig. 5 (a) when $\epsilon$ exceeds $d_{\mathrm{e}}$ and $\rho_{\mathrm{S}}$. The scaling law (21) for $\delta W$ also agrees well, as shown in Fig. 5 (b). While the linear growth rate does depend on the sizes of $d_{\mathrm{e}}$ and $\rho_{\mathrm{S}}\left(\gamma_{\mathrm{L}} \sim \tau_{\mathrm{H}}^{-1} \hat{d}_{\mathrm{e}}^{2 / 3} \hat{\rho}_{\tau}^{5 / 6}\right.$ where $\rho_{\tau}^{2}=\rho_{\mathrm{S}}^{2}+\rho_{\mathrm{i}}^{2}$ according to the linear theory [12]), the explosive nonlinear phase hardly depends on them at all, in agreement with our theory (20).

The effect of resistivity on a case with $\hat{d}_{\mathrm{e}}=\hat{\rho}_{\mathrm{S}}=0.005$
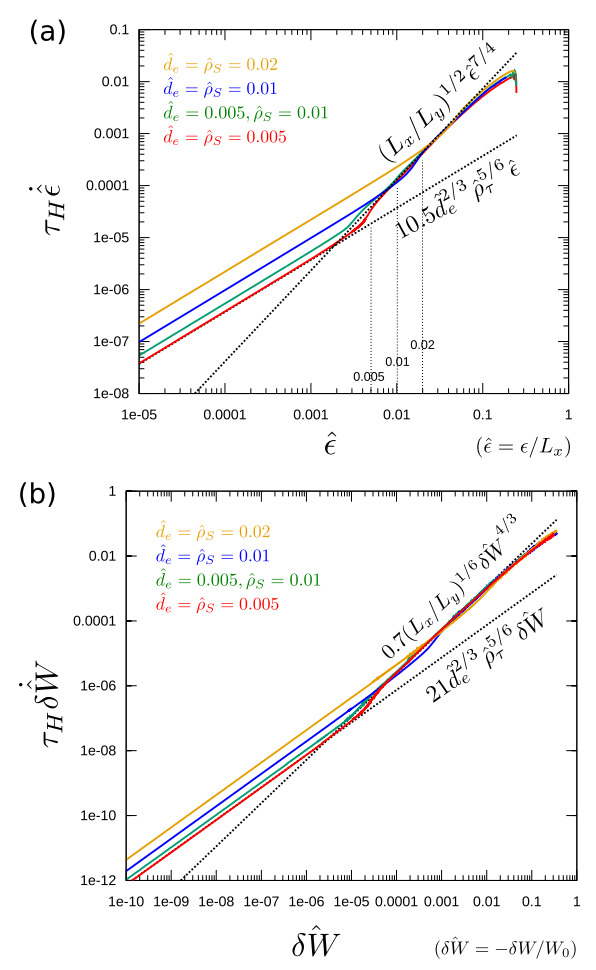

Fig. 5 Explosive scalings for (a) growth of $\epsilon$ and (b) decay of magnetic energy $W$, when $\rho_{\mathrm{i}}=\eta=0$ and $L_{y} / L_{x}=5.84$ $\left(\Delta^{\prime}=534 / L_{x}\right)$. 

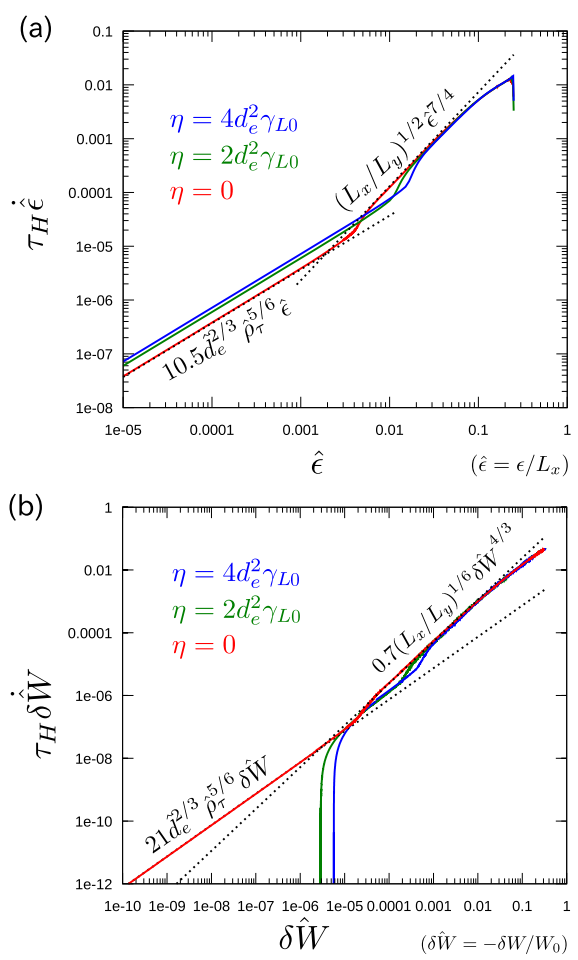

Fig. 6 Explosive scalings for (a) growth of $\epsilon$ and (b) decay of magnetic energy $W$, when $\hat{d}_{\mathrm{e}}=\hat{\rho}_{\mathrm{S}}=0.005, \rho_{\mathrm{i}}=0$ and $L_{y} / L_{x}=5.84\left(\Delta^{\prime}=534 / L_{x}\right)$.

and $\rho_{\mathrm{i}}=\eta=0$ is shown in Fig. 6. In general, let $\gamma_{\mathrm{L} 0}$ be the linear growth rate in the collisionless limit $\eta=0$. One can expect the resistivity to remain negligible so long as the electron-ion collision time $\tau_{\mathrm{e}}=d_{\mathrm{e}}^{2} / \eta$ is much longer than $1 / \gamma_{\mathrm{L} 0}$. On the contrary, if $\eta$ is so large that $\tau_{\mathrm{e}}<1 / \gamma_{\mathrm{L} 0}$, the situation would become more like a resistive (or collisional) reconnection. Figure 6 compares the results of the relatively resistive cases, $\eta=2 d_{\mathrm{e}}^{2} \gamma_{\mathrm{L} 0}$ and $4 d_{\mathrm{e}}^{2} \gamma_{\mathrm{L} 0}$, with that of the collisionless case $\eta=0$. The linear growth rate $\gamma_{\mathrm{L} 0}=10.5 \hat{d}_{\mathrm{e}}^{2 / 3} \hat{\rho}_{\tau}^{5 / 6} / \tau_{\mathrm{H}}$ for $\eta=0$ is indeed enhanced by resistivity. In Fig. 6, the threshold of the transition is elevated by increasing $\eta$, probably because the resistive layer width $\delta_{\eta}\left[\sim\left(\tau_{\mathrm{H}} \eta / k_{y}\right)^{1 / 3}\right.$ in the large- $\Delta^{\prime}$ limit $]$ becomes wider than $d_{\mathrm{e}}=\rho_{\mathrm{S}}$. For these cases, $\delta_{\eta}$ serves as $\delta_{\text {in }}$. However, it is remarkable that the nonlinear phase (where $\epsilon>\delta_{\text {in }}$ ) ends up with the same reconnection speed as the collisionless case $\eta=0$. We have similarly observed a localized Xshaped layer even in the presence of resistivity. Consequently, the widening of the current layer width $\sim \delta_{\text {in }}$ due to resistivity does not seem to affect the reconnection speed in the nonlinear phase. Further, when $\epsilon$ reaches $L_{x} / 4$ in the case with $\eta=4 d_{\mathrm{e}}^{2} \gamma_{\mathrm{L} 0}$, the magnetic energy decreases by $32.7 \%$, or $\delta W=-0.327 W_{0}$. At this time, the kinetic energy is numerically calculated to be $\delta K=0.314 W_{0}$ and the other nonideal-MHD energies, $E_{\mathrm{C}}=(1 / 2) \int d_{\mathrm{e}}^{2}\left|\nabla^{2} \psi\right|^{2} d x d y$ and $E_{\mathrm{T}}=(1 / 2) \int \rho_{\mathrm{S}}^{2}\left|\nabla^{2} \phi\right|^{2} d x d y$, are found to be small $\delta E_{\mathrm{C}}=\delta E_{\mathrm{T}}=0.006 W_{0}$. The total dissipated energy then amounts to $\delta\left(K+W+E_{\mathrm{C}}+E_{\mathrm{T}}\right)=-0.002 W_{0}$. The ideal
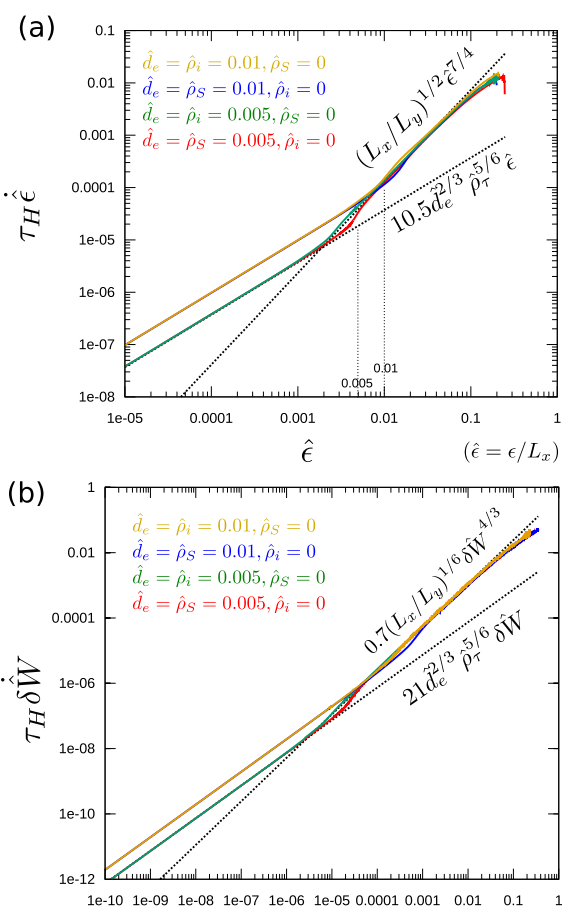

$\delta \hat{W}$

$\left(\delta \hat{W}=-\delta W / W_{0}\right)$

Fig. 7 Explosive scalings for (a) growth of $\epsilon$ and (b) decay of magnetic energy $W$, when $\eta=0$ and $L_{y} / L_{x}=5.84\left(\Delta^{\prime}=\right.$ $\left.534 / L_{x}\right)$.

MHD energy conservation law, $\delta K+\delta W \simeq 0$, thus seems to be well satisfied, even for rather large values of $\eta$.

In Fig. 7, we show the results for a hot-ion case with $d_{\mathrm{e}}=\rho_{\mathrm{i}} \neq 0, \rho_{\mathrm{S}}=0$. These are plotted over the previous hot-electron case with $d_{\mathrm{e}}=\rho_{\mathrm{S}} \neq 0, \rho_{\mathrm{i}}=0$, keeping $\eta=0$. As already remarked in comparing Fig. 3 with Fig. 2, the linear-to-nonlinear transition occurs a little earlier in the hot-ion case. Nevertheless, Fig. 7 shows that the reconnection speed in the nonlinear phase again agrees well with the scalings (20) and (21). In particular, the reconnection speed does not depend on whether electrons or ions are hot.

In the same manner, we have also investigated the reconnection speed in the cold plasma limit $\rho_{\mathrm{S}}=\rho_{\mathrm{i}}=0$. In this case, intermittent acceleration occurs due to the plasmoid instability (see Fig. 1). Our definition of $\epsilon$ is no longer appropriate for such a stochastic nonlinear phase, since it is supposed to parameterize only the coherent growth of a single tearing mode (or a single magnetic island). Hence, we show only the decay rate of the magnetic energy in Fig. 8, for the cases with $\eta=0,2 d_{\mathrm{e}}^{2} \gamma_{\mathrm{L} 0}, 4 d_{\mathrm{e}}^{2} \gamma_{\mathrm{L} 0}$ fixing $\hat{d}_{\mathrm{e}}=0.01$ and $L_{y} / L_{x}=5.24$ (where $\gamma_{\mathrm{L} 0}=8 \hat{d}_{\mathrm{e}}^{2 / 3} / \tau_{\mathrm{H}}$ for $\eta=0$ ). While the decay rate does fluctuate in the nonlinear phase, we find that reconnection tends to be faster than in the linear phase, though always somewhat more slowly than indicated by the scaling law (21). Recall that the plasmoid instability generates "bubbles" of magnetic islands and multiple reconnection points, as shown in Fig. 1, each of which resembles the local reconnection pro- 


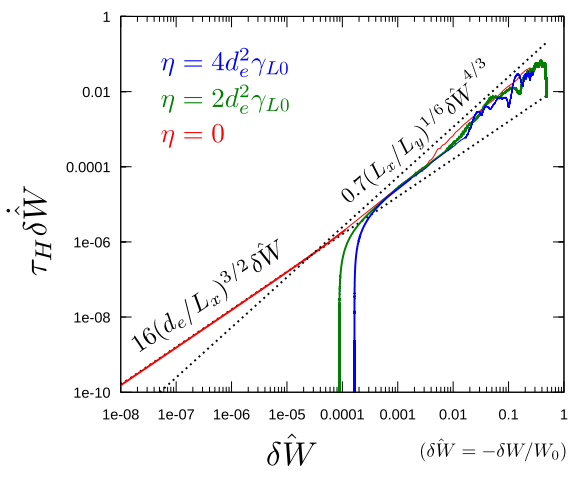

Fig. 8 Explosive scaling for decay of magnetic energy $W$, when $\hat{d}_{\mathrm{e}}=0.01, \hat{\rho}_{\mathrm{S}}=\hat{\rho}_{\mathrm{i}}=0$ and $L_{y} / L_{x}=5.24\left(\Delta^{\prime}=427 / L_{x}\right)$.

cess discussed in Sec. 4. Since the magnetic energy considered here is analogous to the potential energy, it is not surprising that some secondary instability may drive the system to a lower energy state, which is demonstrated to exist in Sec. 4. The reconnection rate shown in Fig. 8, which results from the plasmoid instability, seems to approach the scaling law (21). This is presumably because the plasmoid instability actually triggers local reconnections, releasing magnetic energy more efficiently than the Sweet-Parkerlike global reconnection.

\section{Prediction of Reconnection Time}

Suppose that the reconnection speed makes a transition to that given by Eq. (20) after $\epsilon$ exceeds the inner layer width $\delta_{\text {in }}$. Then we can estimate the reconnection time (or the collapse time) as

$$
\tau_{\text {rec }} \sim \tau_{H}\left(L_{y} / L_{x}\right)^{1 / 2}\left(L_{x} / \delta_{\text {in }}\right)^{3 / 4},
$$

according to the discussion following (9) and (10).

Let us apply this scaling law to the parameters of a solar flare. We choose the density and temperature of the corona region as $n=10^{14} \mathrm{~m}^{-3}$ and $T_{\mathrm{i}}=T_{\mathrm{e}}=2 \times 10^{6} \mathrm{~K}$, respectively. This leads to $d_{\mathrm{e}}=0.53 \mathrm{~m}, \eta=10^{9} T_{\mathrm{e}}^{-3 / 2}=$ $0.35 \mathrm{~m}^{2} / \mathrm{s}$ and $\tau_{\mathrm{e}}=0.8 \mathrm{~s}$.

Although a coronal loop actually has a threedimensional magnetic-field structure, we suppose that the neighborhood of a reconnection point is similar to the geometry we have considered in this paper. The maximum scale of a coronal loop is about $L_{x}=10^{7} \mathrm{~m}$ in width, $L_{y}=10^{8} \mathrm{~m}$ in length, with the peak magnetic field being about $B_{\max }=0.1 \mathrm{~T}$. Using the corresponding Alfvén velocity $v_{\mathrm{A}, \max }=2.2 \times 10^{8} \mathrm{~m} / \mathrm{s}$, we estimate $\tau_{\mathrm{H}} \simeq L_{x} / v_{\mathrm{A}, \max }=0.046 \mathrm{~s}$. Hence the resistive layer width is $\delta_{\eta} \simeq\left(\tau_{\mathrm{H}} \eta / k_{y}\right)^{1 / 3}=64 \mathrm{~m}$. Even though the Lundquist number $S=L_{x} v_{\mathrm{A}, \max } / \eta \sim 10^{15}$ is huge, it follows from the condition $\delta_{\eta}>d_{\mathrm{e}}$ that the resistivity dominates the electron inertia; that is, the reconnection seems to be resistive rather than collisionless.

It is generally difficult to predict the guide field $B_{0}$ around the reconnection point. If we take the average background magnetic field $B_{0}=0.01 \mathrm{~T}$ as the guide field for example, we obtain $\rho_{\mathrm{S}}=\rho_{\mathrm{i}}=0.13 \mathrm{~m}$; these gyroradii are roughly comparable to $d_{\mathrm{e}}$.

Formula (22) requires only the threshold value $\delta_{\text {in }}$, beyond which the explosive growth starts. If we choose the resistive layer width $\delta_{\eta}$ as $\delta_{\text {in }}$, we obtain

$$
\tau_{\text {rec }} \sim 0.3 \text { hour. }
$$

This indicates that our explosive reconnection model is fast enough to explain the observed time scales of solar flares (which range from a few minutes to a few hours).

\section{Summary}

In this work, we have numerically and theoretically investigated whether or not magnetic reconnection occurs explosively in a gyrofluid model. When either the ionsound gyroradius $\rho_{\mathrm{S}}$ or the ion gyroradius $\rho_{\mathrm{i}}$ is comparable to the electron skin depth $d_{\mathrm{e}}$, an explosive magnetic reconnection is triggered from a localized X-shaped current layer, as shown in Fig. 2 and Fig. 3. For different choices of the parameters $\left(\rho_{\mathrm{S}}, \rho_{\mathrm{i}}, d_{\mathrm{e}}\right)$ and even in the presence of a finite resistivity $\eta$, this transition commonly occurs when the mode amplitude $\epsilon$ (or the magnetic island width) exceeds the inner layer width $\delta_{\text {in. }}$. In the subsequent nonlinear phase, we find almost the same ultimate reconnection speed, which agrees well with the explosive scaling laws (20) and (21).

We derived these scaling laws in Sec. 4 in a more sophisticated and general manner than was done in our previous work [15]. In our model, the reconnection speed is simply determined by the ideal-MHD energy balance in the external region (that is, in the whole region except for the current layer), and the magnetic energy is mostly converted into the kinetic energy of the outflow. We are then able to predict explosive reconnection processes by supposing that the $\mathrm{X}$-shaped current layer is localized around the reconnection point. In the strongly nonlinear phase discussed here, the nonideal-MHD effects inside the narrow current layer are not important because the layer width and the energy change within it are negligible in comparison to those in the external region. The explosive reconnection speed therefore does not depend on the sizes of $\rho_{\mathrm{S}}, \rho_{\mathrm{i}}, d_{\mathrm{e}}, \eta$.

In the cold plasma limit $\rho_{\mathrm{S}}=\rho_{\mathrm{i}}=0$, the plasmoid instability occurs instead of the production of a single X-shaped current layer. In this case, the reconnection speed is accelerated intermittently in response to the plasmoid instability, but it never exceeds the explosive scaling law given by (21). We infer that the plasmoid instability triggers local reconnections, enabling a more efficient approach to a lower magnetic energy state. The reconnection speed seems to follow reasonably closely the result given by (21).

By applying the scaling law (20) to the size of a solar flare, we have also shown that the estimated collapse 
time is sufficiently fast to explain the observations. Although the model we have studied here is very simple and our theory is rather heuristic, the explosive reconnection speed given by (20) and (21) is largely independent of the microscale physics inside the current layer and appears to be the fastest one that can be obtained from ideal-MHD energy balance.

\section{Acknowledgments}

This work was supported by JSPS KAKENHI Grant Number 16K05627. Numerical calculations were performed on the UV1000 at the Institute of Fluid Science, Tohoku University.

[1] P.A. Sweet, Electromagnetic Phenomena in Cosmical Physics, IAU Symp. No. 6, edited by B. Lehnert (Cambridge Press, London, 1958) p. 123; E.N. Parker, J. Geophys. Res. 62, 509 (1957).

[2] W.H. Matthaeus and S.L. Lamkin, Phys. Fluids 28, 303 (1985).

[3] D. Biskamp, Phys. Fluids 29, 1520 (1986).

[4] K. Shibata and S. Tanuma, Earth Planets Space 53, 473 (2001).

[5] A. Bhattacharjee, Y.-M. Huang, H. Yang and B. Rogers, Phys. Plasmas 16, 112102 (2009).

[6] L. Comisso, M. Lingam, Y.-M. Huang and A. Bhattacharjee, Phys. Plasmas 23, 100702 (2016).
[7] J. Birn et al., J. Geophys. Res. 106, 3715 (2001).

[8] A.Y. Aydemir, Phys. Fluids B 4, 2469 (1992).

[9] E. Cafaro, D. Grasso, F. Pegoraro, F. Porcelli and A. Saluzzi, Phys. Rev. Lett. 80, 4430 (1998).

[10] D. Grasso, F. Califano, F. Pegoraro and F. Porcelli, Plasma Phys. Rep. 26, 512 (2000).

[11] A. Bhattacharjee, K. Germaschewski and C.S. Ng, Phys. Plasmas 12, 042305 (2005).

[12] L. Comisso, D. Grasso, F.L. Waelbroeck and D. Borgogno, Phys. Plasmas 20, 092118 (2013).

[13] A. Ishizawa and T.-H. Watanabe, Phys. Plasmas 20, 102116 (2013).

[14] H.E. Petschek, Physics of Solar Flares, Edited by W.N. Hess (NASA SP-50, Washington DC, 1964) p.425.

[15] M. Hirota, Y. Hattori and P.J. Morrison, Phys. Plasmas 22, 052114 (2015)

[16] T.J. Schep, F. Pegoraro and B.N. Kuvshinov, Phys. Plasmas 1, 2843 (1994).

[17] E. Tassi, P.J. Morrison, D. Grasso and F. Pegoraro, Nucl. Fusion 50, 034007 (2010).

[18] F.L. Waelbroeck and E. Tassi, Commun. Nonlinear Sci. Numer. Simul. 17, 2171 (2012).

[19] D. Biskamp, Magnetic Reconnection in Plasmas (Cambridge University Press, Cambridge, 2000).

[20] F. Porcelli, Phys. Rev. Lett. 66, 425 (1991).

[21] M. Ottaviani and F. Porcelli, Phys. Rev. Lett. 71, 3802 (1993).

[22] M. Hirota, P.J. Morrison, Y. Ishii, M. Yagi and N. Aiba, Nucl. Fusion 53, 063024 (2013). 